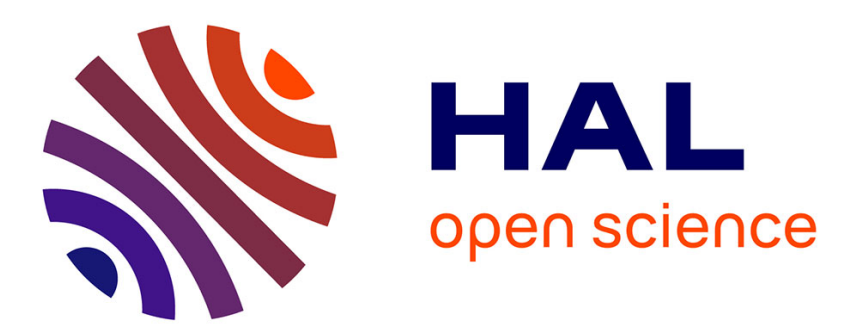

\title{
Uniform-in-Time Convergence of Numerical Schemes for a Two-Phase Discrete Fracture Model
}

\author{
Jerome Droniou, Julian Hennicker, Roland Masson
}

\section{To cite this version:}

Jerome Droniou, Julian Hennicker, Roland Masson. Uniform-in-Time Convergence of Numerical Schemes for a Two-Phase Discrete Fracture Model. FVCA 2017 - International Conference on Finite Volumes for Complex Applications VIII, Jun 2017, Lille, France. 10.1007/978-3-319-57397-7_20 . hal-01541150

\author{
HAL Id: hal-01541150 \\ https://hal.science/hal-01541150
}

Submitted on 25 Jun 2017

HAL is a multi-disciplinary open access archive for the deposit and dissemination of scientific research documents, whether they are published or not. The documents may come from teaching and research institutions in France or abroad, or from public or private research centers.
L'archive ouverte pluridisciplinaire HAL, est destinée au dépôt et à la diffusion de documents scientifiques de niveau recherche, publiés ou non, émanant des établissements d'enseignement et de recherche français ou étrangers, des laboratoires publics ou privés. 


\title{
Uniform-in-time convergence of numerical schemes for a two-phase discrete fracture model
}

\author{
J. Droniou, J. Hennicker, R. Masson
}

\begin{abstract}
Flow and transport in fractured porous media are of paramount importance for many applications such as petroleum exploration and production, geological storage of carbon dioxide, hydrogeology, or geothermal energy. We consider here the two-phase discrete fracture model introduced in [3] which represents explicitly the fractures as codimension one surfaces immersed in the surrounding matrix domain. Then, the two-phase Darcy flow in the matrix is coupled with the two-phase Darcy flow in the fractures using transmission conditions accounting for fractures acting either as drains or barriers. The model takes into account complex networks of fractures, discontinuous capillary pressure curves at the matrix fracture interfaces and can be easily extended to account for gravity including in the width of the fractures. It also includes a layer of damaged rock at the matrix fracture interface with its own mobility and capillary pressure functions. In this work, the convergence analysis carried out in [3] in the framework of gradient discretizations [2] is extended to obtain the uniform-in-time convergence of the discrete solutions to a weak solution of the model.
\end{abstract}

Key words: Discrete fracture model, two-phase Darcy flow, uniform-in-time convergence, gradient discretization method

\footnotetext{
Jérôme Droniou

School of Mathematical Sciences, Monash University, Victoria 3800, Australia, e-mail: jerome. droniou@monash.edu

Julian Hennicker

Université Côte d'Azur, Inria, CNRS, Laboratoire J.A. Dieudonné, team Coffee, France; and CSTJF, TOTAL S.A. - Avenue Larribau, 64018 Pau, France, e-mail: julian.hennickere unice.fr

Roland Masson

Université Côte d'Azur, Inria, CNRS, Laboratoire J.A. Dieudonné, team Coffee, France e-mail: roland.masson@unice.fr.
} 


\section{Continuous model}

We give here a brief overview of the notations, and refer to [3] for more details. $\Omega$ is a bounded polytopal domain of $\mathbb{R}^{d}(d=2,3)$, partitioned into a fracture domain $\Gamma$ and a matrix domain $\Omega \backslash \Gamma$. The network of fractures is $\Gamma=\bigcup_{i \in I} \Gamma_{i}$, where each $\Gamma_{i}$ is planar and has therefore two faces $\mathfrak{a}^{+}(i)$ and $\mathfrak{a}^{-}(i)$. Set $\chi=\left\{\mathfrak{a}^{+}(i), \mathfrak{a}^{-}(i) \mid i \in I\right\}$ the set all faces and write, for simplicity, $\Gamma_{\mathfrak{a}^{+}(i)}=\Gamma_{\mathfrak{a}^{-}(i)}=\Gamma_{i}$. For $\mathfrak{a} \in \chi, \gamma_{\mathfrak{a}}$ is the one-sided trace operator on $\Gamma_{\mathfrak{a}}$ and $\mathbf{n}_{\mathfrak{a}}$ denotes the unit normal vector directed from the face $\mathfrak{a}$ to the matrix domain. The following notations, in which $\bar{u}_{\mu}^{\alpha}$ is the phase pressure in the medium $\mu$ and phase $\alpha$, are used throughout the paper.

$$
\begin{aligned}
& M_{m}=\Omega, M_{f}=\Gamma \text { and } M_{\mathfrak{a}}=\Gamma_{\mathfrak{a}} ; s^{+}=\max (0, s), s^{-}=(-s)^{+} ; \\
& \left(\bar{p}_{m}, \bar{p}_{f}\right)=\left(\bar{u}_{m}^{1}-\bar{u}_{m}^{2}, \bar{u}_{f}^{1}-\bar{u}_{f}^{2}\right) \text { (capillary pressures); } \llbracket \bar{u}^{\alpha} \rrbracket_{\mathfrak{a}}=\gamma_{\mathfrak{a}} \bar{u}_{m}^{\alpha}-\bar{u}_{f}^{\alpha} .
\end{aligned}
$$

The assumptions in the rest of this paper are:

- The matrix-valued functions $\Lambda_{m}$ and $\Lambda_{f}$, permeability tensors in the matrix and fracture domains, respectively, are uniformly coercive tensors.

- The functions $T_{f}$ (half-normal transmissibility in the fracture network), $\phi_{m}$ and $\phi_{f}$ (porosities of the matrix and fracture, respectively), and $d_{f}$ (fracture width) are bounded measurable and uniformly positive.

- The phase mobilities $k_{\mu}^{\alpha}: M_{\mu} \times[0,1] \rightarrow \mathbb{R}$ are bounded uniformly positive Caratheodory functions, $h_{\mu}^{\alpha} \in L^{2}\left((0, T) \times M_{\mu}\right)$ and $\eta>0$.

- The saturation $S_{\mu}^{1}: M_{\mu} \times \mathbb{R} \rightarrow[0,1]$ of the non wetting phase is a Caratheodory function; for a.e. $\mathbf{x} \in M_{\mu}, S_{\mu}^{1}(\mathbf{x}, \cdot)$ is a non-decreasing Lipschitz continuous function on $\mathbb{R} ; S_{\mu}^{1}(\cdot, q)$ is piecewise constant on a finite partition $\left(M_{\mu}^{j}\right)_{j \in J_{\mu}}$ of polytopal subsets of $M_{\mu}$, for all $q \in \mathbb{R}$. Not indicating the phase in the saturation means that $\alpha=1$, that is, $S_{\mu}=S_{\mu}^{1}$. Of course, $S_{\mu}^{2}=1-S_{\mu}^{1}$ The initial capillary pressures $\left(\bar{p}_{m, 0}, \bar{p}_{f, 0}\right)$ belong to $H^{1}(\Omega \backslash \bar{\Gamma}) \times L^{2}(\Gamma)$.

For $\varphi_{\mu} \in L^{2}\left((0, T) \times M_{\mu}\right)$ and a.e. $(t, \mathbf{x}) \in(0, T) \times M_{\mu}$, we let

$$
S_{\mu}^{\alpha}\left(\varphi_{\mu}\right)(t, \mathbf{x})=S_{\mu}^{\alpha}\left(\mathbf{x}, \varphi_{\mu}(t, \mathbf{x})\right) \quad \text { and } \quad[k S]_{\mu}^{\alpha}\left(\varphi_{\mu}\right)(t, \mathbf{x})=k_{\mu}^{\alpha}\left(\mathbf{x}, S_{\mu}^{\alpha}\left(\mathbf{x}, \varphi_{\mu}(t, \mathbf{x})\right)\right) .
$$

The PDEs model writes: find phase pressures $\left(\bar{u}_{m}^{\alpha}, \bar{u}_{f}^{\alpha}\right)$ and velocities $\left(\mathbf{q}_{m}^{\alpha}, \mathbf{q}_{f}^{\alpha}\right)$ $(\alpha=1,2)$, such that

$$
\left\{\begin{aligned}
\phi_{m} \partial_{t} S_{m}^{\alpha}\left(\bar{p}_{m}\right)+\operatorname{div}\left(\mathbf{q}_{m}^{\alpha}\right) & =h_{m}^{\alpha} & & \text { on }(0, T) \times \Omega \backslash \bar{\Gamma} \\
\mathbf{q}_{m}^{\alpha} & =-[k S]_{m}^{\alpha}\left(\bar{p}_{m}\right) \Lambda_{m} \nabla \bar{u}_{m}^{\alpha} & & \text { on }(0, T) \times \Omega \backslash \bar{\Gamma} \\
\phi_{f} d_{f} \partial_{t} S_{f}^{\alpha}\left(\bar{p}_{f}\right)+\operatorname{div}_{\tau}\left(\mathbf{q}_{f}^{\alpha}\right)-\sum_{\mathfrak{a} \in \chi} Q_{f, \mathfrak{a}}^{\alpha} & =d_{f} h_{f}^{\alpha} & & \text { on }(0, T) \times \Gamma \\
\mathbf{q}_{f}^{\alpha} & =-d_{f}[k S]_{f}^{\alpha}\left(\bar{p}_{f}\right) \Lambda_{f} \nabla_{\tau} \bar{u}_{f} & & \text { on }(0, T) \times \Gamma \\
\left.\left(\bar{p}_{m}, \bar{p}_{f}\right)\right|_{t=0} & =\left(\bar{p}_{m, 0}, \bar{p}_{f, 0}\right) & & \text { on }(\Omega \backslash \bar{\Gamma}) \times \Gamma,
\end{aligned}\right.
$$

coupled with the matrix-fracture transmission conditions for all $\mathfrak{a} \in \chi$ 


$$
\left\{\begin{aligned}
\mathbf{q}_{m}^{\alpha} \cdot \mathbf{n}_{\mathfrak{a}}+Q_{f, \mathfrak{a}}^{\alpha} & =\eta \partial_{t} S_{\mathfrak{a}}^{\alpha}\left(\gamma_{\mathfrak{a}} \bar{p}_{m}\right) \\
Q_{f, \mathfrak{a}}^{\alpha} & =[k S]_{f}^{\alpha}\left(\bar{p}_{f}\right) T_{f} \llbracket \bar{u}^{\alpha} \rrbracket_{\mathfrak{a}}^{-}-[k S]_{\mathfrak{a}}^{\alpha}\left(\gamma_{\mathfrak{a}} \bar{p}_{m}\right) T_{f} \llbracket \bar{u}^{\alpha} \rrbracket_{\mathfrak{a}}^{+}
\end{aligned}\right.
$$

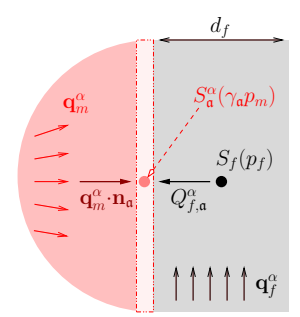

Fig. 1 Illustration of the coupling condition. It can be seen as an upwind two point approximation of $Q_{f, \mathrm{a}}^{\alpha}$. The upwinding takes into account the damaged rocktype of porous thickness $\eta$ at the matrix-fracture interfaces.

To give the weak formulation of this model, set $V^{0}=V_{m}^{0} \times V_{f}^{0}$ with

$$
\begin{aligned}
V_{m}^{0} & =\left\{v \in H^{1}(\Omega \backslash \bar{\Gamma}) \mid \gamma_{\partial \Omega} v=0 \text { on } \partial \Omega\right\}, \\
V_{f}^{0} & =\left\{v \in H^{1}(\Gamma) \mid \gamma_{\partial \Gamma_{i}} v=0 \text { on } \partial \Gamma_{i} \cap \partial \Omega \text { for all } i \in I\right\} .
\end{aligned}
$$

The space $H^{1}(\Gamma)$ is made of functions whose restriction to each $\Gamma_{i}$ belong to $H^{1}\left(\Gamma_{i}\right)$, and whose traces are continuous at fracture intersections $\partial \Gamma_{i} \cap \partial \Gamma_{j}$. Here, $\partial \Gamma_{i}$ is the boundary of $\Gamma_{i}$ respective to the hyperplane containing $\Gamma_{i}$, and $\gamma$ is the trace operator. We abridge $\sum_{\mu \in\{m, f\}}, \sum_{\mathfrak{a} \in \chi}$ and $\sum_{\alpha=1}^{2}$ into, respectively, $\sum_{\mu}, \sum_{\mathfrak{a}}$ and $\sum_{\alpha}$.

Definition 1 (Weak solution of the model). A weak solution of the model is $\left(\bar{u}_{m}^{\alpha}, \bar{u}_{f}^{\alpha}\right)_{\alpha=1,2} \in\left[L^{2}\left(0, T ; V_{m}^{0}\right) \times L^{2}\left(0, T ; V_{f}^{0}\right)\right]^{2}$ such that, for any $\alpha=1,2$ and any $\left(\bar{\varphi}_{m}^{\alpha}, \bar{\varphi}_{f}^{\alpha}\right) \in C_{0}^{\infty}([0, T) \times \Omega) \times C_{0}^{\infty}([0, T) \times \Gamma)$,

$$
\begin{aligned}
& \sum_{\mu}\left(-\int_{0}^{T} \int_{M_{\mu}} \phi_{\mu} S_{\mu}^{\alpha}\left(\bar{p}_{\mu}\right) \partial_{t} \bar{\varphi}_{\mu}^{\alpha} \mathrm{d} \tau_{\mu} \mathrm{d} t+\int_{0}^{T} \int_{M_{\mu}}[k S]_{\mu}^{\alpha}\left(\bar{p}_{\mu}\right) \Lambda_{\mu} \nabla \bar{u}_{\mu}^{\alpha} \cdot \nabla \bar{\varphi}_{\mu}^{\alpha} \mathrm{d} \tau_{\mu} \mathrm{d} t\right. \\
&\left.-\int_{M_{\mu}} \phi_{\mu} S_{\mu}^{\alpha}\left(\bar{p}_{\mu, 0}\right) \bar{\varphi}_{\mu}^{\alpha}(0, \cdot) \mathrm{d} \tau_{\mu}\right)+\sum_{\mathfrak{a}} \int_{0}^{T} \int_{\Gamma_{\mathfrak{a}}} \mathscr{F}\left(\gamma_{\mathfrak{a}} \bar{p}_{m}, \bar{p}_{f}, \llbracket \bar{u}^{\alpha} \rrbracket_{\mathfrak{a}}\right) \llbracket \bar{\varphi}^{\alpha} \rrbracket_{\mathfrak{a}} \mathrm{d} \tau \mathrm{d} t \\
&-\sum_{\mathfrak{a}}\left(\int_{0}^{T} \int_{\Gamma_{\mathfrak{a}}} \eta S_{\mathfrak{a}}^{\alpha}\left(\gamma_{\mathfrak{a}} \bar{p}_{m}\right) \partial_{t} \gamma_{\mathfrak{a}} \bar{\varphi}_{m}^{\alpha} \mathrm{d} \tau \mathrm{d} t+\int_{\Gamma_{\mathfrak{a}}} \eta S_{\mathfrak{a}}^{\alpha}\left(\gamma_{\mathfrak{a}} \bar{p}_{m, 0}\right) \gamma_{\mathfrak{a}} \bar{\varphi}_{m}^{\alpha}(0, \cdot) \mathrm{d} \tau\right) \\
& \quad=\sum_{\mu} \int_{0}^{T} \int_{M_{\mu}} h_{\mu}^{\alpha} \bar{\varphi}_{\mu}^{\alpha} \mathrm{d} \tau_{\mu},
\end{aligned}
$$

where $\mathscr{F}\left(s_{1}, s_{2}, s_{3}\right)=T_{f}\left([k S]_{\mathfrak{a}}^{\alpha}\left(s_{1}\right) s_{3}^{+}-[k S]_{f}^{\alpha}\left(s_{2}\right) s_{3}^{-}\right), \mathrm{d} \tau_{m}(\mathbf{x})=\mathrm{d} \mathbf{x}$ and $\mathrm{d} \tau_{f}(\mathbf{x})=$ $d_{f}(\mathbf{x}) \mathrm{d} \tau(\mathbf{x})(\mathrm{d} \tau$ being the $(d-1)$-dimensional measure on the fractures).

\section{The gradient scheme}

Definition 2 (Gradient Discretization (GD)). A spatial gradient discretisation for a DFN is $\mathscr{D}_{S}=\left(X^{0},\left(\Pi_{\mathscr{D}_{S}}^{\mu}, \nabla_{\mathscr{D}_{S}}^{\mu}\right)_{\mu \in\{m, f\}},\left(\llbracket \llbracket \rrbracket_{\mathfrak{a}, \mathscr{D}_{S}}\right)_{\mathfrak{a} \in \chi},\left(\mathbb{T}_{\mathscr{D}_{S}}^{\mathfrak{a}}\right)_{\mathfrak{a} \in \chi}\right)$, where 
- $X^{0}$ is a finite dimensional space of degrees of freedom,

- $\Pi_{\mathscr{D}_{S}}^{\mu}: X^{0} \rightarrow L^{2}\left(M_{\mu}\right)$ reconstructs a function on $M_{\mu}$ from the DOFs,

- $\nabla_{\mathscr{D}_{S}}^{\mu}: X^{0} \rightarrow L^{2}\left(M_{\mu}\right)^{\operatorname{dim} M_{\mu}}$ reconstructs a gradient on $M_{\mu}$ from the DOFs,

- $\llbracket \cdot \rrbracket_{\mathfrak{a}, \mathscr{D}_{S}}: X^{0} \rightarrow L^{2}\left(\Gamma_{\mathfrak{a}}\right)$ reconstructs, from the DOFs, a jump on $\Gamma_{\mathfrak{a}}$ between the matrix and fracture,

- $\mathbb{T}_{\mathscr{D}_{S}}^{\mathfrak{a}}: X^{0} \rightarrow L^{2}\left(\Gamma_{\mathfrak{a}}\right)$ reconstructs, from the DOFs, a trace on $\Gamma_{\mathfrak{a}}$ from the matrix.

Here, $\Pi_{\mathscr{D}_{S}}^{\mu}$ and $\mathbb{T}_{\mathscr{D}_{S}}^{\mathfrak{a}}$ are piecewise constant reconstructions in the sense of [2], which implies that if $g: \mathbb{R} \rightarrow \mathbb{R}$ then $\Pi_{\mathscr{D}_{S}}^{\mu} g(w)=g\left(\Pi_{\mathscr{D}_{S}}^{\mu} w\right)$ and $\mathbb{T}_{\mathscr{S}_{S}}^{\mathfrak{a}} g(w)=g\left(\mathbb{T}_{\mathscr{D}_{S}}^{\mathfrak{a}} w\right)$. $\mathscr{D}_{S}$ is extended into a space-time GD $\mathscr{D}=\left(\mathscr{D}_{S},\left(\mathrm{I}_{\mathscr{D}}^{\mu}\right)_{\mu \in\{m, f\}},\left(t_{n}\right)_{n=0, \ldots, N}\right)$ with

- $0=t_{0}<t_{1}<\cdots<t_{N}=T$ a discretisation of the time interval $[0, T]$,

- $\mathrm{I}_{\mathscr{D}}^{m}: H^{1}(\Omega \backslash \bar{\Gamma}) \rightarrow X^{0}$ and $\mathrm{I}_{\mathscr{D}}^{f}: L^{2}(\Gamma) \rightarrow X^{0}$ are operators designed to interpolate initial conditions.

The spatial operators are extended into space-time operators the following way. If $w=\left(w_{n}\right)_{n=0, \ldots, N+1} \in\left(X^{0}\right)^{N+1}$, and $\Psi_{\mathscr{D}_{S}}=\Pi_{\mathscr{D}_{S}}^{\mu}, \nabla_{\mathscr{D}_{S}}^{\mu}, \llbracket \cdot \rrbracket_{\mathfrak{a}, \mathscr{D}_{S}}$ or $\mathbb{T}_{\mathscr{D}_{S}}^{\mathfrak{a}}$, then $\Psi_{\mathscr{D} w}$ is defined on $[0, T] \times M_{\mu}$ or $[0, T] \times \Gamma_{\mathfrak{a}}$ by

$\Psi_{\mathscr{D} w}(0, \cdot)=\Psi_{\mathscr{D}_{S}} u_{0}$ and, $\forall n \in\{0, \ldots, N-1\}, \forall t \in\left(t_{n}, t_{n+1}\right] \Psi_{\mathscr{D} w}(t, \cdot)=\Psi_{D_{S}} w_{n+1}$.

We also define the discrete time derivative $\delta_{t} w:(0, T] \rightarrow X^{0}$ by, for the same $n$ and $t$ as above, $\delta_{t} w(t)=\frac{w_{n+1}-w_{n}}{t_{n+1}-t_{n}}$.

The gradient scheme for (2) is: find $\left(u^{\alpha}\right)_{\alpha=1,2} \in\left[\left(X^{0}\right)^{N+1}\right]^{2}$ such that, setting $p=u^{1}-u^{2}$, we have $p_{0}=\left(\mathrm{I}_{\mathscr{D}}^{m} \bar{p}_{m, 0}, \mathrm{I}_{\mathscr{D}}^{f} \bar{p}_{f, 0}\right)$ and, for $\alpha=1,2$ and $v^{\alpha} \in\left(X^{0}\right)^{N+1}$,

$$
\begin{aligned}
& \sum_{\mu} \int_{0}^{T} \int_{M_{\mu}}\left(\phi_{\mu} \Pi_{\mathscr{D}}^{\mu}\left(\delta_{t} S_{\mu}^{\alpha}(p)\right) \Pi_{\mathscr{D}}^{\mu} v^{\alpha}+[k S]_{\mu}^{\alpha}\left(\Pi_{\mathscr{D}}^{\mu} p\right) \Lambda_{\mu} \nabla_{\mathscr{D}}^{\mu} u^{\alpha} \cdot \nabla_{\mathscr{D}}^{\mu} v^{\alpha}\right) \mathrm{d} \tau_{\mu} \mathrm{d} t \\
& +\sum_{\mathfrak{a}}\left(\int_{0}^{T} \int_{\Gamma_{\mathfrak{a}}} \mathscr{F}\left(\mathbb{T}_{\mathscr{D}}^{\mathfrak{a}} p, \Pi_{\mathscr{D}}^{f} p, \llbracket u^{\alpha} \rrbracket_{\mathfrak{a}, \mathscr{D}}\right) \llbracket v^{\alpha} \rrbracket_{\mathfrak{a}, \mathscr{D}} \mathrm{d} \tau \mathrm{d} t\right. \\
& \left.\quad+\int_{0}^{T} \int_{\Gamma_{\mathfrak{a}}} \eta \mathbb{T}_{\mathscr{D}}^{\mathfrak{a}}\left(\delta_{t} S_{\mathfrak{a}}^{\alpha}(p)\right) \mathbb{T}_{\mathscr{D}}^{\mathfrak{a}} v^{\alpha} \mathrm{d} \tau \mathrm{d} t\right)=\sum_{\mu} \int_{0}^{T} \int_{M_{\mu}} h_{\mu}^{\alpha} \Pi_{\mathscr{D}}^{\mu} v^{\alpha} \mathrm{d} \tau_{\mu} \mathrm{d} t .
\end{aligned}
$$

\section{Main result}

Theorem 1. Under the assumptions of Section 1, let $\left(\mathscr{D}^{l}\right)_{l \in \mathbb{N}}$ be a coercive, consistent, limit-conforming and compact sequence of space-time GD (see [3]), and let $\left(u^{\alpha, l}\right)_{l \in \mathbb{N}}$ be such that $u^{\alpha, l} \in\left(X_{l}^{0}\right)^{N_{l}+1}$ is a sequence of solutions of (3) with $\mathscr{D}=\mathscr{D}_{l}$. Then, there exists a weak solution $\left(\bar{u}_{m}^{\alpha}, \bar{u}_{f}^{\alpha}\right)_{\alpha=1,2}$ of the model such that, for all $\mu \in\{m, f\}$ and $\mathfrak{a} \in \chi, S_{\mu}\left(\bar{p}_{\mu}\right):[0, T] \rightarrow L^{2}\left(M_{\mu}\right)$ and $S_{\mathfrak{a}}\left(\gamma_{\mathfrak{a}} \bar{p}_{m}\right):[0, T] \rightarrow L^{2}\left(\Gamma_{\mathfrak{a}}\right)$ are continuous and, up to a subsequence as $l \rightarrow \infty$, with $\bar{p}=\bar{u}^{1}-\bar{u}^{2}$, 


$$
\begin{gathered}
\Pi_{\mathscr{D}}^{\mu} S_{\mu}\left(p^{l}\right) \longrightarrow S_{\mu}\left(\bar{p}_{\mu}\right) \text { in } L^{\infty}\left(0, T ; L^{2}\left(M_{\mu}\right)\right), \\
\mathbb{T}_{\mathscr{D} l}^{\mathfrak{a}} S_{\mathfrak{a}}\left(p^{l}\right) \longrightarrow S_{\mathfrak{a}}\left(\gamma_{\mathfrak{a}} \bar{p}_{m}\right) \text { in } L^{\infty}\left(0, T ; L^{2}\left(\Gamma_{\mathfrak{a}}\right)\right) .
\end{gathered}
$$

Notations and preliminary results. Before proving this theorem, we recall some convergence results established in [3], under the assumptions of Theorem 1. Here, if $\left(w^{l}\right)_{l \in \mathbb{N}}$ is a sequence of functions in $L^{2}((0, T) \times M)$ for some measured space $M$, " $w^{l} \rightarrow w$ in $L^{2}$ " means that the convergence holds in $L^{2}((0, T) \times M)$.

There exists a weak solution $\bar{u}=\left(\bar{u}_{m}, \bar{u}_{f}\right)$ such that, up to a subsequence as $l \rightarrow \infty$, for all $\mu \in\{m, f\}$ and $\mathfrak{a} \in \chi$, with $p=u^{1}-u^{2}$ and $\bar{p}_{\mu}=\bar{u}_{\mu}^{1}-\bar{u}_{\mu}^{2}$,

$$
\begin{aligned}
& \Pi_{\mathscr{D},}^{\mu} u^{\alpha, l} \rightarrow \bar{u}_{\mu}^{\alpha}, \nabla_{\mathscr{D}^{\prime}}^{\mu} u^{\alpha, l} \rightarrow \nabla \bar{u}_{\mu}^{\alpha} \text { and } \llbracket u^{\alpha, l} \rrbracket_{\mathfrak{a}, \mathfrak{Q} l} \rightarrow \llbracket \bar{u}^{\alpha} \rrbracket_{\mathfrak{a}} \text { weakly in } L^{2}, \\
& \Pi_{\mathscr{D}^{\prime}}^{\mu} S_{\mu}\left(p^{l}\right) \rightarrow S_{\mu}\left(\bar{p}_{\mu}\right) \text { and } \mathbb{T}_{\mathscr{D}^{l}}^{\mathfrak{a}} S_{\mathfrak{a}}\left(p^{l}\right) \rightarrow S_{\mathfrak{a}}\left(\gamma_{\mathfrak{a}} \bar{p}_{m}\right) \text { strongly in } L^{2} .
\end{aligned}
$$

The functions $S_{\mu}\left(\bar{p}_{\mu}\right):[0, T] \rightarrow L^{2}\left(M_{\mu}\right)$ and $S_{\mathfrak{a}}\left(\gamma_{\mathfrak{a}} \bar{p}_{m}\right):[0, T] \rightarrow L^{2}\left(\Gamma_{\mathfrak{a}}\right)$ are continuous for the weak topologies of $L^{2}\left(M_{\mu}\right)$ and $L^{2}\left(\Gamma_{\mathfrak{a}}\right)$, respectively. Moreover, for any $\left(T^{l}\right)_{l \in \mathbb{N}} \subset[0, T]$ that converges to some $T^{\infty}$,

$$
\begin{aligned}
& \Pi_{\mathscr{D} l}^{\mu} S_{\mu}\left(p^{l}\right)\left(T^{l}\right) \rightarrow S_{\mu}\left(\bar{p}_{\mu}\right)\left(T^{\infty}\right) \text { weakly in } L^{2}\left(M_{\mu}\right), \text { and } \\
& \mathbb{W}_{\mathscr{D}^{l}}^{\mathfrak{a}} S_{\mathfrak{a}}\left(p^{l}\right)\left(T^{l}\right) \rightarrow S_{\mathfrak{a}}\left(\gamma_{\mathfrak{a}} \bar{p}_{m}\right)\left(T^{\infty}\right) \text { weakly in } L^{2}\left(\Gamma_{\mathfrak{a}}\right) .
\end{aligned}
$$

There exists $\rho_{\mathfrak{a}} \in L^{2}\left((0, T) \times \Gamma_{\mathfrak{a}}\right)$ such that

$$
\mathscr{F}\left(\mathbb{T}_{\mathscr{D} l}^{\mathfrak{a}} p^{l}, \Pi_{\mathscr{D} l}^{f} p^{l}, \llbracket u^{\alpha, l} \rrbracket_{\mathfrak{a}, \mathscr{D} l}\right) \rightarrow \rho_{\mathfrak{a}} \text { weakly in } L^{2},
$$

and, for all $\varphi \in\left[L^{2}\left(0, T ; V_{m}^{0}\right) \times L^{2}\left(0, T ; V_{f}^{0}\right)\right]^{2}$,

$$
\sum_{\alpha, \mathfrak{a}} \int_{0}^{T} \int_{\Gamma_{\mathfrak{a}}} \rho_{\mathfrak{a}} \llbracket \bar{\varphi}^{\alpha} \rrbracket_{\mathfrak{a}} \mathrm{d} \tau \mathrm{d} t=\sum_{\alpha, \mathfrak{a}} \int_{0}^{T} \int_{\Gamma_{\mathfrak{a}}} \mathscr{F}\left(\gamma_{\mathfrak{a}} \bar{p}_{m}, \bar{p}_{f}, \llbracket \bar{u}^{\alpha} \rrbracket_{\mathfrak{a}}\right) \llbracket \bar{\varphi}^{\alpha} \rrbracket_{\mathfrak{a}} \mathrm{d} \tau \mathrm{d} t
$$

For $\rho=\mu \in\{m, f\}$ or $\rho=\mathfrak{a} \in \chi$, let $R_{S_{\rho}(\mathbf{x}, \cdot)}$ be the range of $S_{\rho}(\mathbf{x}, \cdot)$ and $\left[S_{\rho}(\mathbf{x}, \cdot)\right]^{i}: R_{S_{\rho}(\mathbf{x}, \cdot)} \rightarrow \mathbb{R}$ be its pseudo-inverse defined by

$$
\left[S_{\rho}(\mathbf{x}, \cdot)\right]^{i}(q)= \begin{cases}\inf \left\{z \in \mathbb{R} \mid S_{\rho}(\mathbf{x}, z)=q\right\} & \text { if } q>S_{\rho}(\mathbf{x}, 0), \\ 0 & \text { if } q=S_{\rho}(\mathbf{x}, 0), \\ \sup \left\{z \in \mathbb{R} \mid S_{\rho}(\mathbf{x}, z)=q\right\} & \text { if } q<S_{\rho}(\mathbf{x}, 0) .\end{cases}
$$

Let $B_{\rho}(\mathbf{x}, \cdot): \mathbb{R} \rightarrow[0, \infty]$ be given by $B_{\rho}(\mathbf{x}, q)=\int_{S_{\rho}(\mathbf{x}, 0)}^{q}\left[S_{\rho}(\mathbf{x}, \cdot)\right]^{i}(\tau) \mathrm{d} \tau$ if $q \in R_{S_{\rho}(\mathbf{x}, \cdot)}$, $B_{\rho}(\mathbf{x}, q)=+\infty$ otherwise. $B_{\rho}(\mathbf{x}, \cdot)$ is convex 1.s.c. and $B_{\rho}\left(\mathbf{x}, S_{\rho}(\mathbf{x}, \cdot)\right)$ has a subquadratic growth: $B_{\rho}\left(\mathbf{x}, S_{\rho}(\mathbf{x}, r)\right) \leq K r^{2}$ for some $K$ not depending on $\mathbf{x}$ or $r$.

The following continuous (based on [1, Lemma 3.6]) and discrete energy relations hold. For all $T_{0} \in[0, T]$, 


$$
\begin{aligned}
& \sum_{\mu} \int_{M_{\mu}} \phi_{\mu}\left[B_{\mu}\left(S_{\mu}\left(\bar{p}_{\mu}\right)\left(T_{0}\right)\right) \mathrm{d} \tau_{\mu}-\int_{M_{\mu}} \phi_{\mu} B_{\mu}\left(S_{\mu}\left(\bar{p}_{\mu}\right)(0)\right)\right] \mathrm{d} \tau_{\mu} \\
& +\sum_{\mathfrak{a}} \int_{\Gamma_{\mathfrak{a}}}\left[\eta B_{\mathfrak{a}}\left(S_{\mathfrak{a}}\left(\gamma_{\mathfrak{a}} \bar{p}_{m}\right)\left(T_{0}\right)\right) \mathrm{d} \tau_{\mu}-\int_{\Gamma_{\mathfrak{a}}} \eta B_{\mathfrak{a}}\left(S_{\mathfrak{a}}\left(\gamma_{\mathfrak{a}} \bar{p}_{m}\right)(0)\right)\right] \mathrm{d} \tau \\
& +\sum_{\alpha, \mu} \int_{0}^{T_{0}} \int_{M_{\mu}}[k S]_{\mu}^{\alpha}\left(\bar{p}_{\mu}\right) \Lambda_{\mu} \nabla \bar{u}_{\mu}^{\alpha} \cdot \nabla \bar{u}_{\mu}^{\alpha} \mathrm{d} \tau_{\mu} \mathrm{d} t \\
& +\sum_{\alpha, \mathfrak{a}} \int_{0}^{T_{0}} \int_{\Gamma_{\mathfrak{a}}} \mathscr{F}\left(\gamma_{\mathfrak{a}} \bar{p}_{m}, \bar{p}_{f}, \llbracket \bar{u}^{\alpha} \rrbracket_{\mathfrak{a}}\right) \llbracket \bar{u}^{\alpha} \rrbracket_{\mathfrak{a}} \mathrm{d} \tau \mathrm{d} t=\sum_{\alpha, \mu} \int_{0}^{T_{0}} \int_{M_{\mu}} h_{\mu}^{\alpha} \bar{u}_{\mu}^{\alpha} \mathrm{d} \tau_{\mu} \mathrm{d} t
\end{aligned}
$$

and, if $k$ is chosen such that $T_{0} \in\left(t_{k}, t_{k+1}\right]$,

$$
\begin{aligned}
& \sum_{\mu} \int_{M_{\mu}} \phi_{\mu}\left[B_{\mu}\left(S_{\mu}\left(\Pi_{\mathscr{D}_{S}^{l}}^{\mu} p^{l}\right)\left(T_{0}\right)\right)-B_{\mu}\left(S_{\mu}\left(\Pi_{\mathscr{D}_{S}}^{\mu} p_{0}\right)\right)\right] \mathrm{d} \tau_{\mu} \\
& +\sum_{\mathfrak{a}} \int_{\Gamma_{\mathfrak{a}}} \eta\left[B_{\mathfrak{a}}\left(S_{\mathfrak{a}}\left(\mathbb{T}_{\mathscr{D}_{S}^{l}}^{\mathfrak{a}} p^{l}\right)\left(T_{0}\right)\right)-B_{\mathfrak{a}}\left(S_{\mathfrak{a}}\left(\mathbb{T}_{\mathscr{D}_{S}^{l}}^{\mathfrak{a}} p_{0}\right)\right)\right] \mathrm{d} \tau \\
& +\sum_{\alpha, \mu} \int_{0}^{T_{0}} \int_{M_{\mu}}[k S]_{\mu}^{\alpha}\left(\Pi_{\mathscr{D}_{l}}^{\mu} p^{l}\right) \Lambda_{\mu} \nabla_{\mathscr{D}^{l}}^{\mu} u^{\alpha, l} \cdot \nabla_{\mathscr{D}^{l}}^{\mu} u^{\alpha, l} \mathrm{~d} \tau_{\mu} \mathrm{d} t \\
& +\sum_{\alpha, \mathfrak{a}} \int_{0}^{T_{0}} \int_{\Gamma_{\mathfrak{a}}} \mathscr{F}\left(\mathbb{T}_{\mathscr{D} l}^{\mathfrak{a}} p^{l}, \Pi_{\mathscr{D} l}^{f} p^{l}, \llbracket u^{\alpha, l} \rrbracket_{\mathfrak{a}, \mathscr{D} l}\right) \llbracket u^{\alpha, l} \rrbracket_{\mathfrak{a}, \mathscr{D} l} \mathrm{~d} \tau \mathrm{d} t \\
& \leq \sum_{\alpha, \mu} \int_{0}^{t_{k+1}} \int_{M_{\mu}} h_{\mu}^{\alpha} \Pi_{\mathscr{D} l}^{\mu} u^{\alpha, l} \mathrm{~d} \tau_{\mu} \mathrm{d} t .
\end{aligned}
$$

Proof of Theorem 1. The proof follows the ideas initially introduced in [1]. By the characterisation [2, Lemma 4.8] of uniform-in-time convergence, it suffices to prove that, for any sequence $\left(T^{l}\right)_{l \in \mathbb{N}} \subset[0, T]$ converging to some $T^{\infty}$,

$$
\begin{aligned}
& \Pi_{\mathscr{D} l}^{\mu} S_{\mu}\left(p^{l}\right)\left(T^{l}\right) \rightarrow S_{\mu}\left(\bar{p}_{\mu}\right)\left(T^{\infty}\right) \text { in } L^{2}\left(M_{\mu}\right), \\
& \mathbb{\square}_{\mathscr{D} l}^{\mathfrak{d}} S_{\mathfrak{a}}\left(p^{l}\right)\left(T^{l}\right) \rightarrow S_{\mathfrak{a}}\left(\gamma_{\mathfrak{a}} \bar{p}_{m}\right)\left(T^{\infty}\right) \text { in } L^{2}\left(\Gamma_{\mathfrak{a}}\right) .
\end{aligned}
$$

Applying the discrete energy relation (10) to $T_{0}=T^{l}$ yields

$$
\begin{aligned}
& \sum_{\mu} \int_{M_{\mu}} \phi_{\mu} B_{\mu}\left(S_{\mu}\left(\Pi_{\mathscr{D}_{S}^{l}}^{\mu} p^{l}\right)\left(T^{l}\right)\right) \mathrm{d} \tau_{\mu}+\sum_{\mathfrak{a}} \int_{\Gamma_{\mathfrak{a}}} \eta B_{\mathfrak{a}}\left(S_{\mathfrak{a}}\left(\mathbb{T}_{\mathscr{D}_{S}^{l}}^{\mathfrak{a}} p^{l}\right)\left(T^{l}\right)\right) \mathrm{d} \tau \\
& \leq \int_{M_{\mu}} \phi_{\mu} B_{\mu}\left(S_{\mu}\left(\Pi_{\mathscr{D}_{S}}^{\mu} p_{0}\right)\right) \mathrm{d} \tau_{\mu}+\sum_{\mathfrak{a}} \int_{\Gamma_{\mathfrak{a}}} \eta B_{\mathfrak{a}}\left(S_{\mathfrak{a}}\left(\mathbb{T}_{\mathscr{D}_{S}}^{\mathfrak{a}} p_{0}\right)\right) \mathrm{d} \tau \\
& -\sum_{\alpha, \mu} \int_{0}^{T^{l}} \int_{M_{\mu}}[k S]_{\mu}^{\alpha}\left(\Pi_{\mathscr{D}_{l} l}^{\mu} p^{l}\right) \Lambda_{\mu} \nabla_{\mathscr{D} l}^{\mu} u^{\alpha, l} \cdot \nabla_{\mathscr{D} l}^{\mu} u^{\alpha, l} \mathrm{~d} \tau_{\mu} \mathrm{d} t \\
& -\sum_{\alpha, \mathfrak{a}} \int_{0}^{T^{l}} \int_{\Gamma_{\mathfrak{a}}} \mathscr{F}\left(\mathbb{T}_{\mathscr{D} l}^{\mathfrak{a}} p^{l}, \Pi_{\mathscr{D} l}^{f} p^{l}, \llbracket u^{\alpha, l} \rrbracket_{\mathfrak{a}, \mathscr{D} l}\right) \llbracket u^{\alpha, l} \rrbracket_{\mathfrak{a}, \mathscr{D} l} \mathrm{~d} \tau \mathrm{d} t
\end{aligned}
$$




$$
+\sum_{\alpha, \mu} \int_{0}^{t_{k}(l)+1} \int_{M_{\mu}} h_{\mu}^{\alpha} \Pi_{\mathscr{D} l}^{\mu} u^{\alpha, l} \mathrm{~d} \tau_{\mu} \mathrm{d} t=\mathscr{A}_{1}+\mathscr{A}_{2}-\mathscr{A}_{3}-\mathscr{A}_{4}+\mathscr{A}_{5} .
$$

where $k(l)$ is such that $T^{l} \in\left(t_{k(l)}, t_{k(l)+1}\right]$. The consistency of $\left(\mathscr{D}^{l}\right)_{l \in \mathbb{N}}$ shows that $\Pi_{\mathscr{D}_{S}^{\prime}}^{\mu} p_{0}=\Pi_{\mathscr{D}_{S}^{l}}^{\mu} I_{\mathscr{D} l}^{\mu} \bar{p}_{\mu, 0} \rightarrow \bar{p}_{\mu}(0)$ in $L^{2}\left(M_{\mu}\right), \mathbb{T}_{\mathscr{D}_{S}^{\prime}}^{\mathfrak{a}} p_{0}=\mathbb{T}_{\mathscr{D}_{S}^{l}}^{\mathfrak{a}} I_{\mathscr{D} l}^{m} \bar{p}_{m, 0} \rightarrow \gamma_{\mathfrak{a}} \bar{p}_{m}(0)$ in $L^{2}\left(\Gamma_{\mathfrak{a}}\right)$. Since $B_{\rho} \circ S_{\rho}$ is sub-quadratic, we infer

$$
\mathscr{A}_{1}+\mathscr{A}_{2} \rightarrow \int_{M_{\mu}} \phi_{\mu} B_{\mu}\left(S_{\mu}\left(\bar{p}_{\mu}(0)\right)\right) \mathrm{d} \tau_{\mu}+\sum_{\mathfrak{a}} \int_{\Gamma_{\mathfrak{a}}} \eta B_{\mathfrak{a}}\left(S_{\mathfrak{a}}\left(\gamma_{\mathfrak{a}} \bar{p}_{m}(0)\right)\right) \mathrm{d} \tau
$$

The convergence of $\mathscr{A}_{5}$ is trivial from the weak convergence of $\Pi_{\mathscr{D}}^{\mu} u^{\alpha, l}$ and the fact that $t_{k(l)+1} \rightarrow T^{\infty}$ :

$$
\mathscr{A}_{5} \rightarrow \sum_{\alpha, \mu} \int_{0}^{T^{\infty}} \int_{M_{\mu}} h_{\mu}^{\alpha} \bar{u}_{\mu}^{\alpha} \mathrm{d} \tau_{\mu} \mathrm{d} t
$$

Consider Lemma 1 applied to $F^{l}((t, \mathbf{x}), \xi)=\mathbf{1}_{\left(0, T^{l}\right)}(t)[k S]_{\mu}^{\alpha}\left(\Pi_{\mathscr{D} l}^{\mu} p^{l}\right)(t, \mathbf{x}) \Lambda_{\mu}(\mathbf{x}) \xi$ and $W^{l}=\nabla_{\mathscr{D}}^{\mu} u^{\alpha, l}$. By (4) and (5), $W^{l} \rightarrow W:=\nabla \bar{u}_{\mu}^{\alpha}$ weakly in $L^{2}\left((0, T) \times M_{\mu}\right)$ and, up to a subsequence, $\mathbf{1}_{\left(0, T^{l}\right)} \Pi_{\mathscr{D}^{l}}^{\mu} S_{\mu}\left(p^{l}\right) \Lambda_{\mu} \rightarrow \mathbf{1}_{\left(0, T^{\infty}\right)}[k S]_{\mu}^{\alpha}\left(\bar{p}_{\mu}\right) \Lambda_{\mu}$ a.e. on $M_{\mu} \times(0, T)$ while remaining bounded. Since $F^{l}$ is monotonic with respect to its second argument, the assumptions of Lemma 1 are satisfied with $\rho=\mathbf{1}_{\left(0, T^{\infty}\right)}[k S]_{\mu}^{\alpha}\left(\bar{p}_{\mu}\right) \Lambda_{\mu} \nabla \bar{u}_{\mu}^{\alpha}$, and therefore

$$
\liminf _{l \rightarrow \infty} \mathscr{A}_{3} \geq \sum_{\alpha, \mu} \int_{0}^{T^{\infty}} \int_{M_{\mu}}[k S]_{\mu}^{\alpha}\left(\bar{p}_{\mu}\right) \Lambda_{\mu} \nabla \bar{u}_{\mu}^{\alpha} \cdot \nabla \bar{u}_{\mu}^{\alpha} \mathrm{d} \tau_{\mu} \mathrm{d} t .
$$

To study the limit of $\mathscr{A}_{4}$, we apply again Lemma 1 , this time with $F^{l}((t, \mathbf{x}), \xi)=$ $\mathscr{F}\left(\mathbb{T}_{\mathscr{D} /}^{\mathfrak{a}} l^{l}(t, \mathbf{x}), \Pi_{\mathscr{D} l}^{f} p^{l}(t, \mathbf{x}), \xi\right)$ and $W^{l}=\llbracket u^{\alpha, l} \rrbracket_{\mathfrak{a}, \mathscr{D}^{l}}$. From the definition of $\mathscr{F}$ it can be readily checked that $F^{l}$ is monotonic with respect to its first argument. Using therefore the strong convergences (5) of $S_{\mathfrak{a}}^{\alpha}\left(\mathbb{T}_{\mathscr{g} l}^{\mathfrak{a}} p^{l}\right)$ and $S_{\mu}\left(\Pi_{\mathscr{D} l}^{f} p^{l}\right)$, the weak convergence (4) of $\llbracket u^{\alpha, l} \rrbracket_{\mathfrak{a}, \mathscr{D}^{l}}$ and the convergence property (7)-(8) of $F^{l}\left(\cdot, W^{l}\right)=$ $\mathscr{F}\left(\mathbb{T}_{\mathscr{D} l}^{\mathfrak{a}} p^{l}, \Pi_{\mathscr{D} l}^{f} l^{l}, \llbracket u^{\alpha, l} \rrbracket_{\mathfrak{a}, \mathscr{D} l}\right)$, the assumptions of Lemma 1 are satisfied and

$$
\liminf _{l \rightarrow \infty} \mathscr{A}_{4} \geq \sum_{\alpha, \mathfrak{a}} \int_{0}^{T} \int_{\Gamma_{\mathfrak{a}}} \mathscr{F}\left(\gamma_{\mathfrak{a}} \bar{p}_{m}, \bar{p}_{f}, \llbracket \bar{u}^{\alpha} \rrbracket_{\mathfrak{a}}\right) \llbracket \bar{u}^{\alpha} \rrbracket_{\mathfrak{a}} \mathrm{d} \tau \mathrm{d} t .
$$

Gathering (13), (14), (15) and (16) into (12) and using the energy equality (9) yields

$$
\begin{aligned}
\limsup _{l \rightarrow \infty} & \left(\sum_{\mu} \int_{M_{\mu}} \phi_{\mu} B_{\mu}\left(S_{\mu}\left(\Pi_{\mathscr{D}_{S}^{l}}^{\mu} p^{l}\right)\left(T^{l}\right)\right) \mathrm{d} \tau_{\mu}+\sum_{\mathfrak{a}} \int_{\Gamma_{\mathfrak{a}}} \eta B_{\mathfrak{a}}\left(S_{\mathfrak{a}}\left(\mathbb{T}_{\mathscr{D}_{S}}^{\mathfrak{a}} p^{l}\right)\left(T^{l}\right)\right) \mathrm{d} \tau\right) \\
\leq & \sum_{\mu} \int_{M_{\mu}} \phi_{\mu} B_{\mu}\left(S_{\mu}\left(\bar{p}_{\mu}\right)\left(T^{\infty}\right)\right) \mathrm{d} \tau_{\mu}+\sum_{\mathfrak{a}} \int_{\Gamma_{\mathfrak{a}}} \eta B_{\mathfrak{a}}\left(S_{\mathfrak{a}}\left(\bar{p}_{f}\right)\left(T^{\infty}\right)\right) \mathrm{d} \tau
\end{aligned}
$$


On the other hand, the weak $L^{2}$ convergences (6) and the fact that the functions $B_{\rho}$ are convex lower semi-continuous give, by [1, Lemma 3.4],

$$
\begin{aligned}
& \sum_{\mu} \int_{M_{\mu}} \phi_{\mu} B_{\mu}\left(S_{\mu}\left(\bar{p}_{\mu}\right)\left(T^{\infty}\right)\right) \mathrm{d} \tau_{\mu} \leq \liminf _{l \rightarrow \infty} \sum_{\mu} \int_{M_{\mu}} \phi_{\mu} B_{\mu}\left(S_{\mu}\left(\Pi_{\mathscr{D}_{S}^{l}}^{\mu} p^{l}\right)\left(T^{l}\right)\right) \mathrm{d} \tau_{\mu} \\
& \sum_{\mathfrak{a}} \int_{\Gamma_{\mathfrak{a}}} \eta B_{\mathfrak{a}}\left(S_{\mathfrak{a}}\left(\bar{p}_{f}\right)\left(T^{\infty}\right)\right) \mathrm{d} \tau \leq \liminf _{l \rightarrow \infty} \sum_{\mathfrak{a}} \int_{\Gamma_{\mathfrak{a}}} \eta B_{\mathfrak{a}}\left(S_{\mathfrak{a}}\left(\mathbb{T}_{\mathscr{D}_{S}^{l}}^{\mathfrak{a}} p^{l}\right)\left(T^{l}\right)\right) \mathrm{d} \tau .
\end{aligned}
$$

Combining (17), (18) and (19) yields, by [2, Lemma 4.33],

$$
\begin{aligned}
\sum_{\mu} \int_{M_{\mu}} \phi_{\mu} B_{\mu}\left(S_{\mu}\left(\bar{p}_{\mu}\right)\left(T^{\infty}\right)\right) \mathrm{d} \tau_{\mu} & =\lim _{l \rightarrow \infty} \sum_{\mu} \int_{M_{\mu}} \phi_{\mu} B_{\mu}\left(S_{\mu}\left(\Pi_{\mathscr{D}_{S}}^{\mu} p^{l}\right)\left(T^{l}\right)\right) \mathrm{d} \tau_{\mu} \\
\sum_{\mathfrak{a}} \int_{\Gamma_{\mathfrak{a}}} \eta B_{\mathfrak{a}}\left(S_{\mathfrak{a}}\left(\bar{p}_{f}\right)\left(T^{\infty}\right)\right) \mathrm{d} \tau & =\lim _{l \rightarrow \infty} \sum_{\mathfrak{a}} \int_{\Gamma_{\mathfrak{a}}} \eta B_{\mathfrak{a}}\left(S_{\mathfrak{a}}\left(\mathbb{T}_{\mathscr{D}_{S}^{l}}^{\mathfrak{a}} p^{l}\right)\left(T^{l}\right)\right) \mathrm{d} \tau .
\end{aligned}
$$

The proof of (11), and thus of Theorem 1, is then completed using the exact same reasoning as in [1, Section 4.3].

Lemma 1 (Weak Fatou by monotonicity). Let $k \geq 1, M$ be a measured space, and let $\left(F^{l}\right)_{l \in \mathbb{N}}$ be Caratheodory functions $M \times \mathbb{R}^{k} \rightarrow \mathbb{R}^{k}$ such that, for a.e. $\mathbf{z} \in M$ and all $\xi, \eta \in \mathbb{R}^{k},\left[F^{l}(\mathbf{z}, \xi)-F^{l}(\mathbf{z}, \eta)\right] \cdot[\xi-\eta] \geq 0$. Let $\left(W^{l}\right)_{l \in \mathbb{N}}$ such that, as $l \rightarrow \infty, W^{l} \rightarrow$ $W$ weakly in $L^{2}(M)^{k},\left(F^{l}(\cdot, W)\right)_{l \in \mathbb{N}}$ converges strongly in $L^{2}(M)^{k}$, and $F^{l}\left(\cdot, W^{l}\right) \rightarrow$ $\rho$ weakly in $L^{2}(M)^{k}$. Then $\int_{M} \rho(\mathbf{z}) \cdot W(\mathbf{z}) \mathrm{d} \mathbf{z} \leq \liminf _{l \rightarrow \infty} \int_{M} F^{l}\left(\mathbf{z}, W^{l}(\mathbf{z})\right) \cdot W^{l}(\mathbf{z}) \mathrm{d} \mathbf{z}$.

Proof. We have $\left[F^{l}\left(\mathbf{z}, W^{l}\right)-F^{l}(\mathbf{z}, W)\right] \cdot\left[W^{l}-W\right] \geq 0$. Integrate and develop:

$$
0 \leq \int_{M} F^{l}\left(\mathbf{z}, W^{l}\right) \cdot W^{l} \mathrm{~d} \mathbf{z}-\int_{M} F^{l}\left(\mathbf{z}, W^{l}\right) \cdot W \mathrm{~d} \mathbf{z}+\int_{M} F^{l}(\mathbf{z}, W) \cdot\left[W^{l}-W\right] \mathrm{d} \mathbf{z} .
$$

The last term goes to 0 by strong convergence of $F^{l}(\cdot, W)$ and weak convergence of $W^{l}$. By weak convergence of $F^{l}\left(\cdot, W^{l}\right)$, the second term goes to $\int_{M} \rho \cdot W$. The proof is concluded by taking the inferior limit of (20).

Acknowledgements We thank TOTAL S.A. and the Australian Research Council's Discovery Projects funding scheme (project number DP170100605) for partially supporting this work.

\section{References}

1. Droniou, J., Eymard, R.: Uniform-in-time convergence of numerical methods for non-linear degenerate parabolic equations. Numer. Math. 132(4), 721-766 (2016). DOI 10.1007/ s00211-015-0733-6. URL http://dx.doi.org/10.1007/s00211-015-0733-6

2. Droniou, J., Eymard, R., Gallouët, T., Guichard, C., Herbin, R.: The gradient discretisation method: A framework for the discretisation and numerical analysis of linear and nonlinear elliptic and parabolic problems (2016). URL https: / / hal . archives-ouvertes. fr/ hal- 01382358

3. Droniou, J., Hennicker, J., Masson, R.: Numerical analysis of a two-phase flow discrete fracture model URL https: / / arxiv.org/abs/1612.07373. Submitted 